04

\title{
Регистрация собственного вращения пылевых частиц в условиях ВЧ разряда индукционного типа
}

\author{
() В.Ю. Карасев, Е.С. Дзлиева, С.И. Павлов, Л.А. Новиков, И.Ч. Машек \\ Санкт-Петербургский государственный университет, \\ 199034 Санкт-Петербург, Россия \\ e-mail: plasmadust@yandex.ru, v.karasev@spbu.ru
}

(Поступило в Редакцию 13 февраля 2018 г.)

\begin{abstract}
Исследована динамика собственного вращения (вокруг центра масс) пылевых частиц в магнитном поле. Впервые проведены измерения угловой скорости собственного вращения в пылевой ловушке в разряде ВЧ типа. Измеренная угловая скорость вращения не обнаруживает зависимости от магнитной индукции до значений $700 \mathrm{G}$, ожидающейся от действия ионного увлечения. Также впервые измерена скорость собственного вращения в зависимости от давления газа разряда во время нахождения частиц в пылевой ловушке и от вкладываемой в разряд мощности. Экспериментальные результаты хорошо согласуются с развиваемой моделью появления собственного вращения пылевой частицы в процессе поддержания ее стационарного заряда.
\end{abstract}

DOI: 10.21883/JTF.2019.01.46961.71-18

\section{Введение}

Пылевая плазма [1-3] сегодня является не только отдельным типом плазмы, но и системой, в которой реализуются и изучаются необычные для вещества физические свойства [4-10]. В отличие от механических, термодинамических и электрических свойств, которые в пылевой плазме за последние 20 лет изучались в различной степени, магнитные свойства остаются не изученными практически полностью. Касательно экспериментальных работ в этом контексте, можно говорить только о $[11,12]$ и серии продолжающихся исследований [13-16]. Здесь мы не затрагиваем проблему динамики поведения плазменно-пылевых структур во внешнем магнитном поле, которая для слабых магнитных была решена в предыдущие 10 лет [17-25], а для сильных магнитных полей решается в настоящее время [26-30].

Для изучения магнитных свойств пылевой плазмы, создаваемой из частиц немагнитных материалов, нужно рассматривать систему быстро вращающихся сильно заряженных волчков размера в десятки микрон. Вращаясь с частотой порядка $10^{3} \mathrm{~Hz}$ и неся на поверхности заряд до $10^{6} e$, волчки обладают магнитным моментом величиной до $10^{5} \mu_{\mathrm{B}}$.

Находясь в плазме, пылевые частицы приобретают собственное вращение вокруг центра инерции. Принципиальными вопросами для данной задачи являются следующие: выяснение причины и механизма раскручивания волчка, зависимость угловой скорости от магнитного поля, ориентации магнитных моментов (угловых скоростей) системы волчков.

В ряде теоретических работ, например [31-35], причиной вращения предполагается действие направленного потока плазмы (тока разряда, потока из-за неоднородности условий и геометрии) в магнитном поле или без него. В исследовании [16] мы пытались найти влияние такого потока на угловую скорость частиц (влияние кластера соседних частиц, роль асимметричного положения в пылевой ловушке). Но убедительным ответом на данный вопрос было бы экспериментальное исследование собственного вращения частиц в ВЧ разряде, где отсутствует прямой ток. Литературные данные показывают, что ранее экспериментальные исследования собственного вращения проводились только в условиях разряда постоянного тока.

В настоящей работе ставится задача провести поисковое исследование собственного вращения пылевых частиц в условиях ВЧ разряда. Необходимо подобрать условия для реализации эксперимента, определить, существует ли вращение в отсутствие магнитного поля, и получить зависимость скорости вращения от магнитной индукции при максимально возможном ее значении.

\section{Эксперимент и результаты}

Для создания магнитного поля с индукцией до 1 T использовался электромагнит, создающий между полюсами диаметром $30 \mathrm{~cm}$, разнесенными на дистанцию $5 \mathrm{~cm}$, горизонтально направленное магнитное поле с высокой степенью однородности $0.01 \%$ во всем внутреннем объеме. Для эксперимента в качестве разрядной камеры была выбрана разрядная трубка диаметром 2 и длиной $50 \mathrm{~cm}$ и ВЧ разряд индукционного типа. Трубка с малым перпендикулярным отростком, в котором располагался контейнер с пылевыми частицами, не была снабжена электродами и зондами, имела плоское торцевое окно для наблюдения с высоким оптическим увеличением без значительных аберраций. На боковую поверхность трубки была намотана антенна ВЧ генератора, состоящая из трех витков медного провода (рис. 1). В части экспериментов трубка ориентировалась внутри магнитных полюсов горизонтально, в части вертикально. 


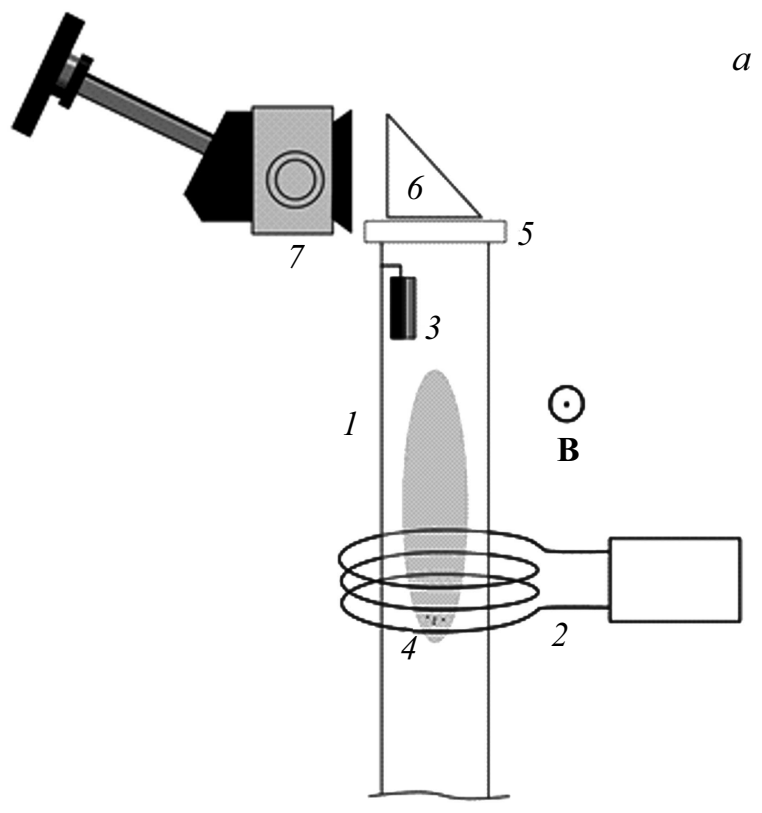

$b$

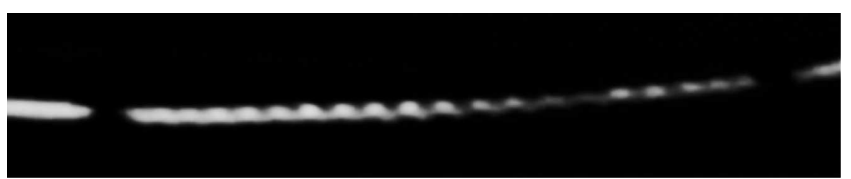

Рис. 1. $a-$ схема экспериментальной установки по наблюдению собственного вращательного движения частиц в ВЧ разряде индукционного типа. 1 - стеклянная трубка, 2 - ВЧ генератор, 3 - контейнер с пылевыми частицами, 4 - пылевая ловушка, 5 - торцевой окно, 6 поворотная призма, 7 - оптический микроскоп с системой видеорегистрации. В - пример развертки пылевой частицы. Условия: азот, $p=0.05 \mathrm{~mm} \mathrm{Hg}, U_{p}=300 \mathrm{~V}, a=7 \mu \mathrm{m}$, кадр развертки демонстрирует 21 модуляцию, что соответствует частоте собственного вращения $630 \mathrm{~Hz}$.

Вкладываемая в разряд мощность оценена как $60 \%$ от мощности, подаваемой на генератор и определяемой током источника и значением напряжения $U_{p}$, частота генератора $100 \mathrm{MHz}$. Трубка через систему вакуумных кранов и натекателя соединялась с вакуумной системой и термопарным измерителем давления. В работе использовался азот, при горении разряда давление газа поддерживалось постоянным, либо задавалась скорость его изменения.

Для создания пылевой компоненты применялись частицы борсиликатного стекла размером от 10 до $120 \mu \mathrm{m}$ с плотностью $0.4-0.9 \mathrm{~g} / \mathrm{cm}^{3}$ пустотелые внутри. На рис. 1 показана одна из реализаций геометрии эксперимента, который проводился следующим образом. При выбранном давлении зажигался ВЧ разряд, имеющий форму плазмоида длиной от 5 до $10 \mathrm{~cm}$ в зависимости от мощности и давления газа. Далее вбрасывались частицы путем встряхивания контейнера, имеющего сетчатое дно, они подсвечивались излучением лазера мощностью
$20 \mathrm{~mW}$ так, что расширенный пучок высвечивал всю область падения или левитации частиц в ловушке. Частицы левитировали вблизи нижнего края плазмоида в области сильного электрического поля. С помощью оптической системы, выполненной на базе длиннофокусного немагнитного микроскопа, производилось наблюдение образов частиц и запись на видеокамеру. В созданных условиях: газ азот, давление $0.01-0.1 \mathrm{~mm} \mathrm{Hg}$ левитация частиц оказалась возможной в магнитном поле до $400 \mathrm{G}$ при горизонтально ориентированной разрядной трубке, и до $700 \mathrm{G}$ в вертикально ориентированной трубке. Последнее связано с изменением направления действия силы термофореза в балансе сил.

В представляемых первых экспериментах условия существования пылевой плазмы подбирались эмпирически. В используемой разрядной трубке количественная диагностика не производилась. Для количественных оценок параметры плазмы брались из литературных данных [36-39], а также оценивались по балансу сил для левитирующих частиц наблюденного размера. Температура электронов была принята $T_{e}=5 \mathrm{eV}$, заряд частиц радиуса $5 \mu \mathrm{m} q=4 \cdot 10^{4} e$, масса частицы $m_{d}=5 \cdot 10^{-13} \mathrm{~kg}$, напряженность электрического поля в области левитации оценена, как $E=6 \mathrm{~V} / \mathrm{cm}$.

Наблюдения образов частиц в микроскоп во время из левитации в плазме показали, что в условиях эксперимента левитируют микросферы диаметром $10 \mu \mathrm{m}$, а также осколки микросфер большего размера. В отсутствие магнитного поля они имеют угловую скорость собственного вращения от 500 до $10000 \mathrm{rad} / \mathrm{s}$. Измерение угловой скорости производилось по методу координатной развертки, детально описанному ранее $[13,14,16]$. При увеличении магнитного поля в области $100 \mathrm{G}$ плазмоид (и пылевая ловушка) проходят область нестабильности. При выпадении частиц производился их новый доброс, и измерения продолжались от значения магнитной индукции $200 \mathrm{G}$. Зависимость угловой скорости собственного вращения от магнитного поля для нескольких частиц показана на рис. 2. На рис. 3 приведен фрагмент зависимости угловой скорости одной из частиц при изменении давления газа разряда. Уменьшение давления производилось достаточно медленно так, что частица не выпадала, и все время находилась в пылевой ловушке в области наблюдения с высоким оптическим увеличением. На рис. 4 приведен пример зависимости угловой скорости собственного вращения от напряжения источника (вкладываемой мощности).

\section{Качественная интерпретация}

Среди полученных результатов отсутствие зависимости угловой скорости собственного вращения от магнитной индукции является неожиданным. Элементарные оценки импульса, получаемого пылевой частицей от ионного потока, как принято в пылевой плазме, уравновешенного импульсом, передаваемым нейтральному 


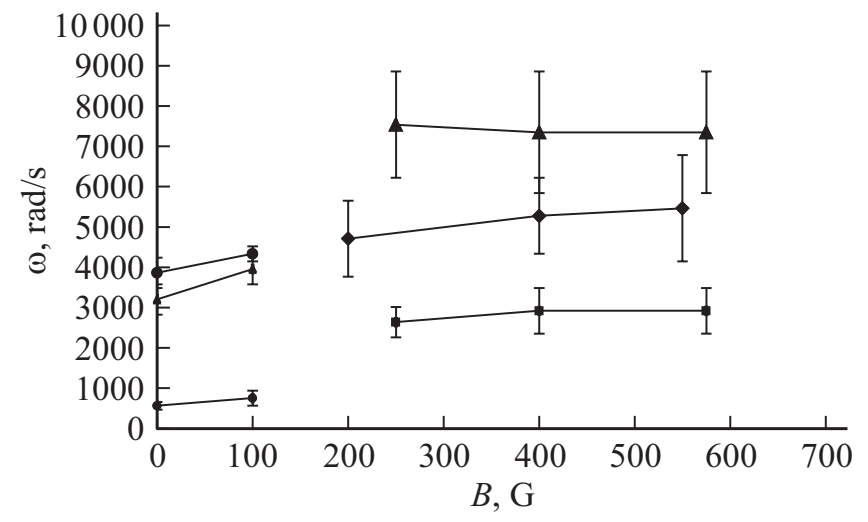

Рис. 2. Зависимость угловой скорости собственного вращения пылевых частиц от величины магнитного поля в пылевой ловушке в ВЧ разряде. Условия: азот, $p=0.05-0.08 \mathrm{~mm} \mathrm{Hg}$, частицы - полые стеклянные микросферы диаметром $10 \mu \mathrm{m}$. Частота ВЧ генератора $100 \mathrm{MHz}$, подаваемое напряжение $350 \mathrm{~V}$. В области $150 \mathrm{G}$ плазмоид меняет конфигурацию, после $200 \mathrm{G}$ измерения продолжаются с новыми частицами.

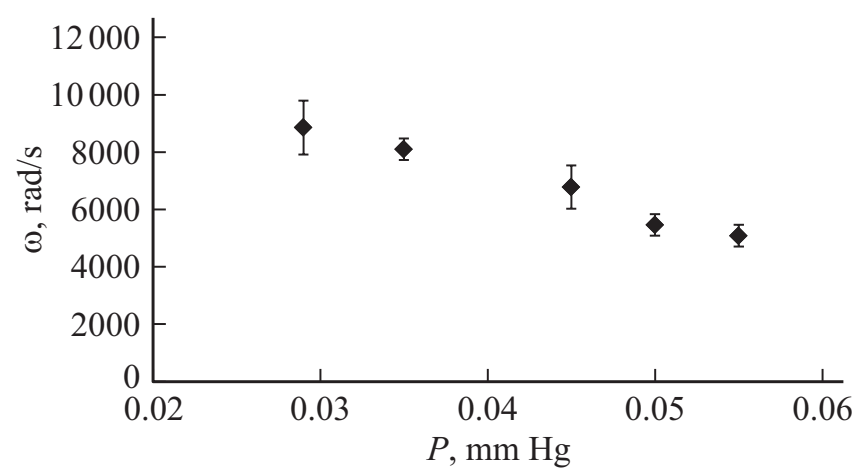

Рис. 3. Зависимость угловой скорости собственного вращения пылевых частиц от давления в ВЧ разряде. Условия: азот, $a=7 \mu \mathrm{m}$. Частота ВЧ генератора $100 \mathrm{MHz}$, подаваемое напряжение $300 \mathrm{~V}$.

газу из-за трения при вращении, дают угловую скорость порядка $10^{5} \mathrm{rad} / \mathrm{s}$. Теоретические модели, развиваемые в [34,35], предсказывают еще больший порядок скорости $10^{6} \mathrm{rad} / \mathrm{s}$. Зависимости скорости вращения от давления и мощности разряда оказались ожидаемыми. Обсудим полученные результаты по порядку.

Проведем количественную интерпретацию угловой скорости собственного вращения пылевой частицы в магнитном поле. В условиях эксперимента при $p \leq 0.1 \mathrm{~mm} \mathrm{Hg}$ дебаевский радиус экранирования значительно меньше длины пробега плазменных частиц. В процессе поддержания стационарного заряда на поверхность пылевой частицы непрерывно идут ионы и электроны с длины дебаевского масштаба в бесстолкновительном режиме. Рассмотрим сферическую частицу радиусом $a=5 \mu \mathrm{m}$, она находится под плавающим потенциалом $U_{\mathrm{fl}}$ и имеет отрицательный заряд $q=a U_{\mathrm{fl}}$. Достигающие ее поверхности ионы ускоряются полем частицы и получают скорость значительно больше тепловой, порядка $10^{4} \mathrm{~m} / \mathrm{s}$. Только самые быстрые электроны способны преодолеть потенциальный барьер и достигнуть поверхности частицы.

В стационарном случае потоки электронов и ионов на поверхность частицы должны быть равными $n_{e} v_{e}=n_{i} v_{i}$. Стационарность поверхностного заряда частицы (мы не рассматриваем флуктуации заряда из-за дискретности процесса зарядки $[5,7,8])$ требует, чтобы средние скорости движения электронов и ионов к частице были равными $\left\langle v_{e}\right\rangle=\left\langle v_{i}\right\rangle$. Это означает, что при своем движении ионы и электроны в перпендикулярном магнитном поле будут приобретать одинаковый азимутальный импульс. Поскольку сила $F_{\mathrm{L}}=e v_{r} B$ не зависит от массы частицы, а электроны и ионы проходят в процессе поддержания стационарного заряда равные расстояния за равные времена, то азимутальная составляющая импульса у них у поверхности частицы будет одинаковой. Рекомбинация на поверхности - неупругий процесс, можно предположить, что приобретенная азимутальная составляющая импульса полностью передается частице. В итоге, процесс поддержания стационарного заряда пылевой частицы в магнитном поле не приведет к раскручиванию частицы вокруг ее цента инерции.

Представленное качественное рассмотрение может объяснить отсутствие зависимости угловой скорости вращения от магнитного поля (см. рис. 2), а также аналогичное отсутствие зависимости, полученное ранее в тлеющем разряде $[14,15]$. Заметим, что рассмотрение потоков плазменных частиц без учета влияния магнитного поля в данном его диапазоне справедливо, так как электронные коэффициенты диффузии и подвижности остаются большими, чем ионные. Поскольку ионы не замагничены $\omega_{i} \tau_{i}<1$ ( $\omega_{i}$ - циклотронная частота, $\tau_{i}-$ время между столкновениями ионов), то для них коэффициент диффузии в магнитном поле не изменяется. Для электронов $\omega_{e} \tau_{e}>1$, но еще выполняется условие $D_{i} \ll D_{e} /\left(1+\omega_{e}^{2} \tau_{e}^{2}\right)$, где $D_{i}$ и $D_{e}-$ коэффициенты диффузии ионов и электронов в отсутствие магнитного поля. Изменение потока электронов можно ожидать только в магнитном поле около $1 \mathrm{~T}$, когда циклотронный радиус электрона будет сравним с размером самой пылевой частицы (или зонда) [40,41].

Пример зависимости угловой скорости собственного вращения от давления является уникальной возможностью проверить данную зависимость непосредственно (на одной частице). Согласно развиваемому представлению [13-16], угловая скорость слабо асимметричных частиц с формой, близкой к сферической, может быть представлена в виде

$$
\omega=\frac{9 \eta e n_{i} q_{d} k T_{n}}{2 \pi \varepsilon_{0} p m_{n} V_{T n} a^{2}},
$$

где $V_{T n}-$ тепловая скорость газа, $p$ - давление газа, $n_{i}$ - концентрация ионов, $\varepsilon_{0}-$ электрическая постоянная, $k-$ постоянная Больцмана, $\eta-$ степень 


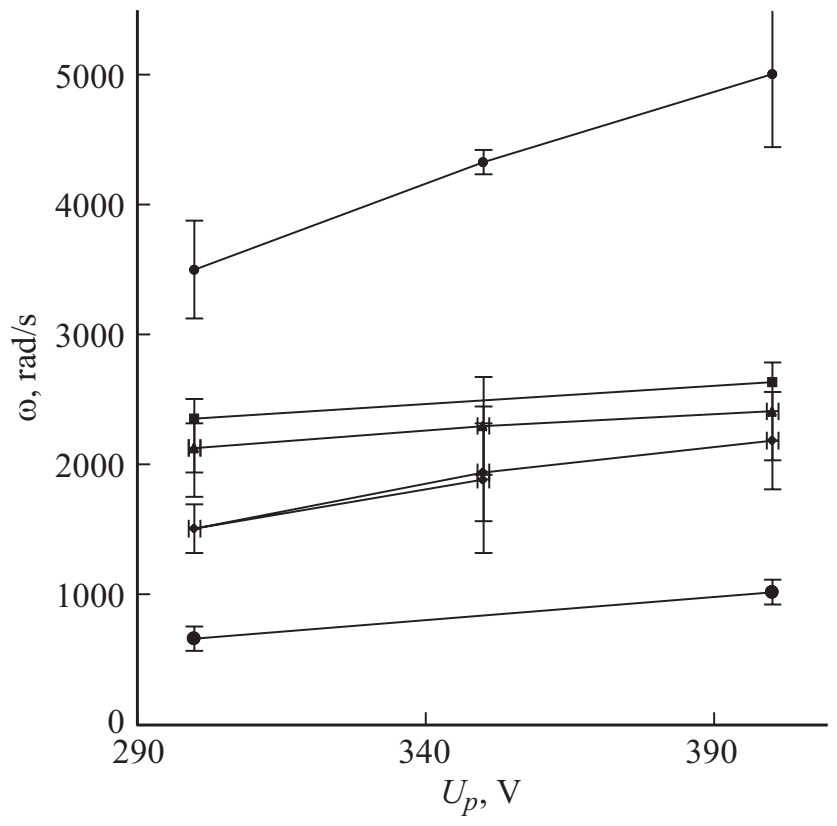

Рис. 4. Зависимость угловой скорости уединенных пылевых частиц от величины $U_{p}$. Условия: азот, $p=0.05-0.11 \mathrm{~mm} \mathrm{Hg}$, частицы - полые стеклянные микросферы радиусом 5-7 $\mu \mathrm{m}$.

асимметрии частицы. Сопоставление с зависимостью на рис. 3 дает хорошее согласие.

Увеличение напряжения $U_{p}$, дающее увеличение мощности (и соответственное увеличение плотности плазмы $\left.n_{i}\right)$, вызывает увеличение угловой скорости, показанное на рис. 4 , что также согласуется с приведенной формулой. Количественные соответствия эксперимента с численной оценкой получаются, если предполагать величину неидеальности геометрии частицы $\eta$ до $20 \%$, как это выполнялось для условий тлеющего разряда [13]. Таким образом, полученные в ВЧ разряде зависимости качественно согласуются с моделью раскручивания пылевого волчка, получающего азимутальный импульс от плазменных частиц в процессе поддержания стационарного заряда.

\section{Заключение}

В настоящей работе впервые проведены измерения угловой скорости собственного вращения пылевых частиц в пылевой ловушке в ВЧ разряде. Впервые измерена угловая скорость вращения от магнитной индукции вплоть до значений $700 \mathrm{G}$, которая не обнаруживает изменения, ожидаемого от действия ионного увлечения. Измерены зависимости скорости вращения от давления газа разряда во время нахождения частицы в пылевой ловушке и от вкладываемой мощности. Зависимости хорошо согласуются с развиваемой моделью появления собственного вращения частицы в процессе поддержания ее стационарного заряда.
Эксперимент частично выполнен при поддержке РФФИ 14-02-00313, интерпретация проведена в рамках проекта РФФИ 18-02-00113.

\section{Список литературы}

[1] Chu J., Lin I. // Phys. Rev. Lett. 1994. P. 724009.

[2] Thomas H., Morfill G., Demmel V., Goree J., Feuerbacher B., Mohlmann D. // Phys. Rev. Lett. 1994. Vol. 73. P. 652.

[3] Hayashi Y., Tachibana K. // Jpn. J. Appl. Phys. 1994. P. 33L804.

[4] Bouchoule A. Dusty Plasmas: Physics, Chemistry, and Technological Impact in Plasma Processing. NY.: John Wiley \& Sons, 1999. 408 p.

[5] Fortov V.E., Mofill G.E. Complex and dusty plasmas: from laboratory to space. NY:: Taylor \& Francis Group, 2010. $440 \mathrm{p}$.

[6] Shukla P.K., Mamun A.A. Introduction to Dusty Plasma Physics. Bristol: Institute of Physics Publishing, 2002. 395 p.

[7] Vladimirov S.V., Ostrikov K., Samarian A.A. Physics and Applications of Complex Plasmas. London: Imperial College Press, 2005. 439 p.

[8] Tsytovich V.N., Morfill G.E., Vladimirov S.V., Thomas H.M. Elementary Physics of complex plasmas. NY.: Springer, 2008. $370 \mathrm{p}$.

[9] Карасев В.Ю., Дзлиева Е.С., Павлов С.И. Лабораторная пылевая плазма в магнитном поле. СПб.: Свое Издательство, 2016. 136 с.

[10] Ваулина О.С., Петров О.Ф., Фортов В.Е., Храпак А.Г., Храпак C.A. Пылевая плазма эксперимент и теория. М.: Физматлит, 2009. 315 с.

[11] Sato N. // AIP Conf. Proc. 2005. Vol. 799. P. 97.

[12] Samsonov D., Zhdanov S., Morfill G. // New Journ. Phys. 2003. Vol. 5. P. 24.

[13] Karasev V.Yu., Dzlieva E.S., Eikhval'd A.I. et al. // Phys. Rev. E. 2009. Vol. 79. P. 026406.

[14] Karasev V.Yu., Dzlieva E.S., Ermolenko M.A., Golubev M.S., Ivanov A.Yu. // Contr. Plasma Phys. 2011. Vol. 51. P. 509.

[15] Дзлиева Е.С., Карасев В.Ю., Петров О.Ф. // ЖЭТФ. 2012. T. 141. № 1. C. 189.

[16] Карасев В.Ю., Ермоленко М.А., Дзлиева Е.С., Павлов С.И., Новиков Л.А., Машек И.Ч. // ЖТФ. 2016. Т. 86. C. $139-144$.

[17] Дзлиева E.C., Карасев В.Ю., Эйхвальд А.И. // Опт. и спектр. 2002. Т. 92. № 6. С. 1018-1023.

[18] Karasev V.Yu., Dzlieva E.S., Ivanov A.Yu., Eikhval'd A.I. // Phys. Rev. E. 2006. Vol. 74. N 6. P. 066403.1-12.

[19] Васильев М.М., Дьячков Л.Г., Антипов С.Н., Петров О.Ф., Фортов В.Е. // Письма в ЖЭТФ. 2007. Т. 86. № 6. C. $414-419$.

[20] Дзлиева Е.С., Ермоленко М.А., Карасев В.Ю., Павлов С.И., Новиков Л.А., Майоров С.А. // Письма в ЖЭТФ. 2014. T. 100. № 11. C. 801-806.

[21] Nedospasov A.V. // Phys. Rev. E. 2009. Vol. 79. P. 036401.

[22] Nedospasov A.V. // EPL. 2013 Vol. 103. P. 25001.

[23] Vasiliev M.M., D'yachkov L.G., Antipov S.N., Huijink R., Petrov O.F., Fortov V.E. // EPL. 2011. Vol. 93. P. 15001.1-6.

[24] Dzlieva E.S., Karasev V.Yu., Pavlov S.I. // EPL. 2015. Vol. 110. P. 55002.

[25] Дзлиева Е.С., Карасев В.Ю., Машек И.Ч., Павлов С.И. // ЖТФ. 2016. Т. 86. C. 145-148. 
[26] Sato N. // AIP Conf. Proc. 2002. Vol. 649. P. 66.

[27] Schwabe M., Konopka U., Morfill G.E. et al. // Phys. Rev. Lett. 2011. Vol. 106. P. 215004.

[28] Thomas E.Jr., Lynch B., Konopka U., Merlino R.L., Rosenberg M. // Phys. Plasmas. 2015. Vol. 22. P. 030701.1-4.

[29] Thomas E. Jr., Konopka U., Lynch B., Adams S., Le Blanc S., Merlino R.L., Rosenberg M. // Phys. Plasmas. 2015. Vol. 22. P. 113708.

[30] Karasev V.Yu., Dzlieva E.S., Pavlov S.I., Novikov L.A., Maiorov S.A. // IEEE Trans. Plasma. Sci. 2017. Vol. PP. N 99. P. 1-4.

[31] Hutchinson I.H. // New Jour. Phys. 2004. Vol. 6. P. 43.1.

[32] Ishihara O., Sato N. // IEEE Trans. Plasma. Sci. 2001. Vol. 29. P. 179.

[33] Tsytovich V., Vladimirov S. // IEEE Trans. on Plasma Sci. 2004. Vol. 32. P. 659.

[34] Stepanenko A.A., Krasheninnikov S.I. // Phys. Plasm. 2013. Vol. 20. P. 033702.

[35] Krasheninnikov S.I. // Phys. Plasma. 2006. Vol. 13. P. 114502.

[36] Райзер Ю.П. Физика газового разряда. М.: Наука, 1992. $536 \mathrm{c}$.

[37] Fortov V.E., Usachev A.D., Zobnin A.V., Petrov O.F. // Phys. Rev. E. 2004. Vol. 70. P. 046415.

[38] Fortov V.E., Usachev A.D., Zobnin A.V., Molotkov V.I., Petrov O.F. // Phys. Plasma. 2003. Vol. 10. P. 1199.

[39] Zobnin A.V., Usachev A.D., Fortov V.E. // AIP Conf. Proc. 2002. Vol. 649. P. 293.

[40] Chen F.F. Electric probes. Plasma diagnostic techniques. NY: Academic Press, 1965. $200 \mathrm{p}$.

[41] Chan P., Talbot L., Turian K. Electrical Probes in Stationary and Flowing Plasmas, Theory and Application. Berlin, Heidelberg, NY.: Springer-Verlag, 1975. 197 p. 